\title{
Common primary tumours of the abdomen and pelvis and their patterns of tumour spread as seen on multi-detector computed tomography
}

\author{
Nyree Griffin • Christopher Burke • \\ Lee Alexander Grant
}

Received: 13 November 2010/Revised: 9 January 2011 /Accepted: 14 March 2011 / Published online: 14 April 2011

(C) European Society of Radiology 2011

\begin{abstract}
Background Multidetector computed tomography (MDCT) has become the main investigation of choice for staging of many cancers.

Aim The purpose of this pictorial review is to discuss the imaging appearances on CT of some of the more common cancers arising within the abdomen and pelvis and to describe their typical sites of local, nodal and haematogenous tumour spread.

Methods Cancers arising from the stomach, pancreas, colon, kidney, ovary and prostate will be reviewed.

Results Awareness of the characteristic sites of tumour spread is important to allow accurate identification of all sites of disease.

Conclusion This will clearly have an impact on both patient management and prognosis.
\end{abstract}

Keywords Multidetector CT · Abdomen · Pelvis ·

Neoplasm · Metastases

\section{Introduction}

In the last 2 decades, cross-sectional imaging, in particular multidetector computed tomography (MDCT), has become

N. Griffin • C. Burke

Department of Radiology,

Guys' and St Thomas' NHS Foundation Trust,

London, UK

L. A. Grant $(\bowtie)$

Department of Radiology, The Royal Free Hospital,

Pond Street,

London NW3 2QG, UK

e-mail: leegrant100@gmail.com the main investigation of choice in the locoregional and distant staging of many tumours within the abdomen and pelvis. MDCT benefits from having a high spatial resolution and fast acquisition time, making it an ideal tool to stage and restage tumours. The routine use of intravenous (and oral contrast) media, unless contraindicated, also helps to delineate sites of disease, especially within solid organs. The identification of nodal involvement on $\mathrm{CT}$ is based on nodal size, typically using a minimum cutoff of $1 \mathrm{~cm}$ in short axis diameter, although this can lead to both underand over-staging of metastatic nodal involvement. The purpose of this pictorial review is to discuss the imaging appearances on $\mathrm{CT}$ of some of the more common cancers arising within the abdomen and pelvis and to describe their typical sites of local, nodal and haematogenous tumour spread. Cancers arising from the stomach, pancreas, colon, kidney, ovary and prostate will be reviewed, with summary tables provided. Lymphoma will not be specifically discussed.

\section{Gastric cancer}

Ninety percent of tumours are adenocarcinomas, which are subdivided into two main histological types: (1) the welldifferentiated (intestinal) type, which is associated with atrophic gastritis, occurs in older patients and has a better survival rate and (2) the undifferentiated (diffuse/signet ring) type, which is more common, occurs more frequently in women and has a poorer prognosis [1]. Gastric adenocarcinoma occurs either proximally (cardia) or distally (non-cardia), with the former increasing in incidence whilst the latter is declining (see Table 1).

On CT, the use of oral contrast agents to distend the stomach is essential, preferably with water rather than dilute gastrograffin to avoid beam-hardening artefacts. Involvement 
Table 1 Summary table of local, nodal and haematogenous spread in gastric cancer

\begin{tabular}{ll}
\hline Local spread & - Local spread into adjacent structures (e.g. \\
& pancreas, colon, spleen) \\
Lymph node & Perigastric: pericardial; lesser curvature; greater \\
spread & curvature; suprapyloric \\
& $\cdot$ Extraperigastric: left gastric; common hepatic; \\
& coeliac; splenic hilum and artery; hepatic \\
& pedicle; retropancreatic; mesenteric root; middle \\
& colic; para-aortic \\
Haematogenous & - Most commonly the liver (25\% at presentation) \\
spread & and peritoneum \\
Notes & Transcoelomic spread can occur through the \\
& peritoneum (e.g. Kruckenberg tumours) \\
& Retropancreatic, para-aortic and mesenteric \\
& nodes are classified as M1 metastatic disease \\
& Nodal disease in GIST tumours is extremely rare \\
\hline
\end{tabular}

of the stomach wall is seen as thickening or irregularity of the inner/middle layer of the gastric wall (T1 tumours) or transmural thickening with abnormal contrast enhancement (T2 tumours) [2]. Wall thickness has been reported to be greater and of higher attenuation in the diffuse histological type compared to the intestinal type [3]. Gastric cancer spreads in a predictable fashion through the gastric wall with the presence of perigastric fat stranding and nodular outline suggestive of serosal involvement (T3 tumours) (Fig. 1). Direct invasion into adjacent organs and structures indicates T4 tumours.

Spread to perigastric and less frequently other intraabdominal nodes may occur (Table 1; Fig. 1). Troisier's sign describes the clinical finding of an enlarged left supraclavicular lymph node due to metastatic involvement.

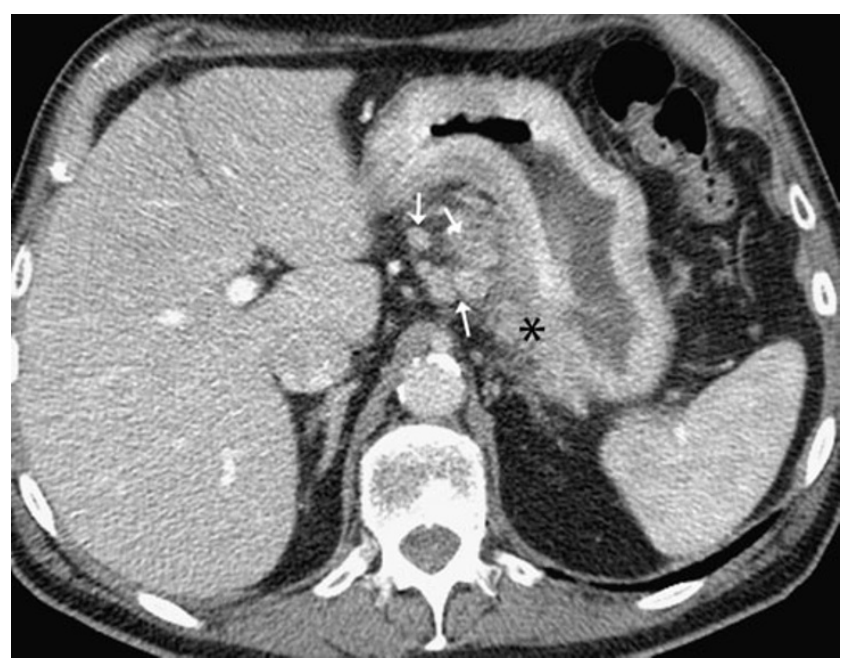

Fig. 1 Gastric adenocarcinoma: axial contrast-enhanced CT (CECT) showing tumour arising from the lesser curvature of the stomach (asterisk) associated with enlarged regional gastrohepatic nodes (arrows)
Abdominal cancers may metastasise to this site via the thoracic duct, and gastric malignancy is classically described as the commonest primary tumour to do this. Obstructive jaundice with associated bile duct dilatation may occur, due to enlarged porta hepatis nodes or due to the spread of gastric tumour via the gastrohepatic ligament directly into the liver.

Haematogeneous spread via the portal vein to the liver occurs in $25 \%$ of patients at presentation, with liver metastases typically appearing as rim-enhancing lowattenuation lesions during the portal venous phase. Peritoneal spread occurs in a similar percentage at presentation. Peritoneal disease secondary to gastric adenocarcinoma may mimic the appearance of peritoneal disease secondary to metastatic ovarian carcinoma, with omental cake and/or discrete peritoneal deposits noted within the abdominal cavity. Trans-coelomic spread is also characteristic of gastric cancer, with spread to the ovary resulting in a Krukenberg tumour that typically appears as a mixed solid/cystic adnexal mass. Involvement of the peritoneal reflection within the pelvis can result in a positive Blumer's shelf finding, with tumour found high on the anterior rectal wall [4].

It should be noted that other histological tumour types arising from the stomach have different patterns of tumour spread. Gastrointestinal stromal tumours (GISTs) are the most common primary mesenchymal neoplasms of the gastrointestinal tract, distinct from true smooth muscle and neural tumours, and are derived from the interstitial cells of Cajal. They are most frequently located within the stomach $(60$ $70 \%$ ) and should be regarded as having malignant potential. GISTs typically involve the outer muscular layer of the GI tract, and thus often demonstrate exophytic growth (Fig. 2). Most appear as well-defined extraluminal or combined intraluminal/extraluminal masses on CT. If small, they tend to be of homogeneous attenuation; if larger, they are more heterogeneous on both the unenhanced and enhanced scans because of necrosis or haemorrhage [5]. The central areas of necrosis may communicate with the gastric lumen. Displacement of adjacent structures is more often seen than direct invasion. If arising from the stomach, the tumour may extend into the gastrohepatic ligament, the gastrosplenic ligament and the lesser sac. Nodal spread is extremely rare, but haematogenous spread can occur, often to the liver with nearly $50 \%$ of patients with GISTs presenting with metastases (Fig. 2) [5]. Peritoneal seeding is also seen. Less commonly, metastases to the soft tissue, lungs and pleura may occur. Metastatic deposits have similar CT characteristics to the primary GIST [6].

Pancreatic cancer

Pancreatic ductal adenocarcinoma accounts for more than $80 \%$ of malignant tumours of the pancreas, and $70 \%$ of 


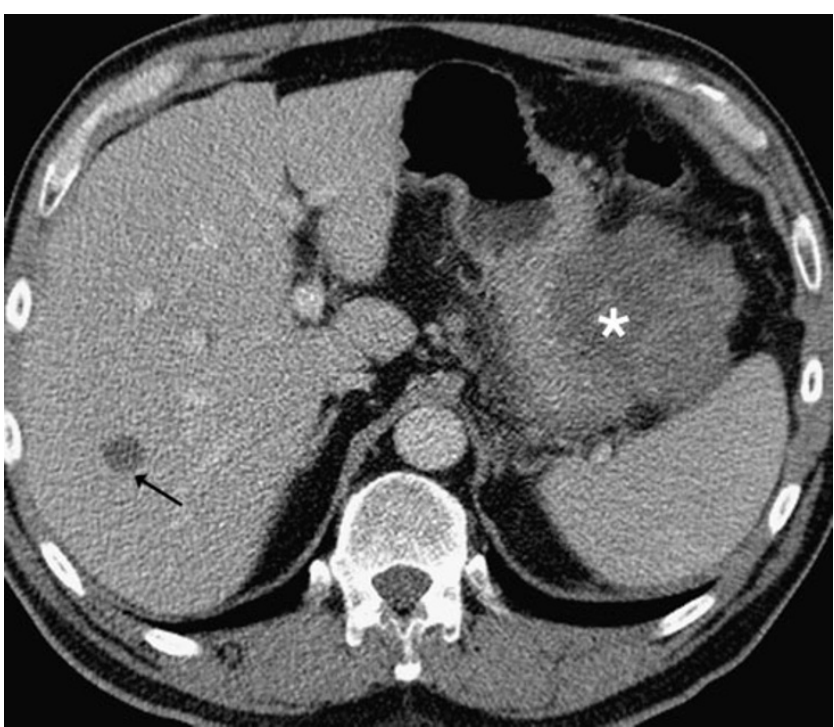

Fig. 2 Gastrointestinal stromal tumour (GIST): axial CECT showing typical large low attenuation exophytic tumour (asterisk) arising from the greater curvature of the stomach associated with liver metastasis (arrow). Note there are no significantly enlarged regional nodes

tumours arise within the pancreatic head. CT is the initial imaging modality used for staging of suspected pancreatic cancer with CT (along with MRI and endoscopic sonography) used to distinguish between potentially resectable and non-resectable patients. For cancer at the head of the pancreas, curative surgery involves a pancreaticoduodenectomy (Whipple procedure). Contraindications to surgery include distant metastases, circumferential involvement of the superior mesenteric vein-portal vein segment more than $2 \mathrm{~cm}$ long, thrombus in the vein, and occlusion or circumferential invasion of the celiac, hepatic or superior mesenteric arteries [7] (see Table 2).

Table 2 Summary table of local, nodal and haematogenous spread in pancreatic cancer

$\begin{array}{cc}\text { - Tocal spread } & \text { perineural invasion; } \\ & \text { - Local invasion can involve the stomach, } \\ \text { duodenum and retroperitoneum; } \\ \text { • Head/uncinate process tumours: these usually } \\ \text { extend along the SMA and mesenteric root; } \\ \text { • Body/tail tumours: these usually infiltrate the } \\ \text { celiac, hepatic or splenic arteries } \\ \text { • Primary drainage: superior, inferior, anterior, } \\ \text { posterior and splenic lymph nodes; } \\ \text { - Secondary drainage: porta hepatis, common } \\ \text { spread } \\ \text { hepatic, coeliac, mesenteric root lymph nodes; } \\ \text { - Tertiary drainage: peri-aortic and distal superior } \\ \text { mesenteric lymph nodes } \\ \text { - These usually involve the liver and peritoneal } \\ \text { surfaces } \\ \text { Haematogenous } \\ \text { spread }\end{array}$
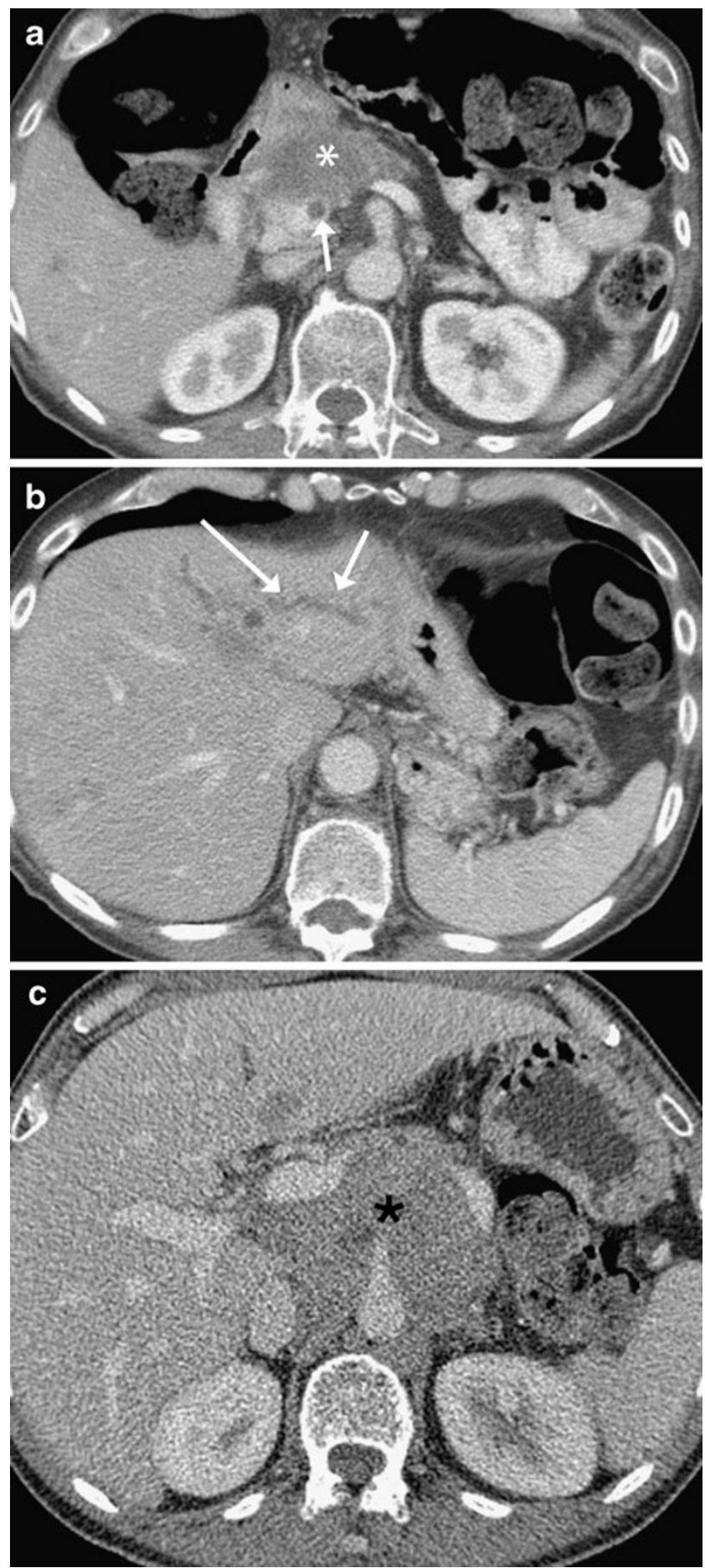

Fig. 3 Pancreatic adenocarcinoma: a axial CECT showing an example of a tumour arising in the head of the pancreas (asterisk) with invasion into the duodenum. There is a focal thrombus in the portal vein (arrow); b axial CECT in same patient as (a); there is associated intra- (arrows) and extrahepatic (not shown) bile duct dilatation; c axial CECT showing a large bulky tumour in a different patient arising in the body of the pancreas (asterisk) encasing the origin of the celiac axis 
When performing a staging CT, ideally at least two contrast-enhanced acquisitions (during the late arterial and venous phases) are advised. Again oral contrast with water is helpful to allow delineation of the duodenal wall.

The pancreas is a retroperitoneal organ and has a close anatomic relationship with the abdominal peritoneal reflections. The pancreatic head is connected to the liver and lesser curvature of the stomach via the hepatoduodenal ligament and gastrohepatic ligaments, respectively. The tail of the pancreas is in continuity with the hilum of the spleen and the greater curvature of the stomach via the splenorenal and gastrosplenic ligaments. Pancreatic cancer can invade these adjacent peritoneal ligaments (as well as the transverse mesocolon) to involve the stomach, duodenum (Fig. 3a) and retroperitoneum [8]. Tumour tends to spread by direct perivascular and perineural invasion within the subperitoneal space [8]. If arising from the pancreatic head or uncinate process, tumours usually extend along the superior mesenteric artery
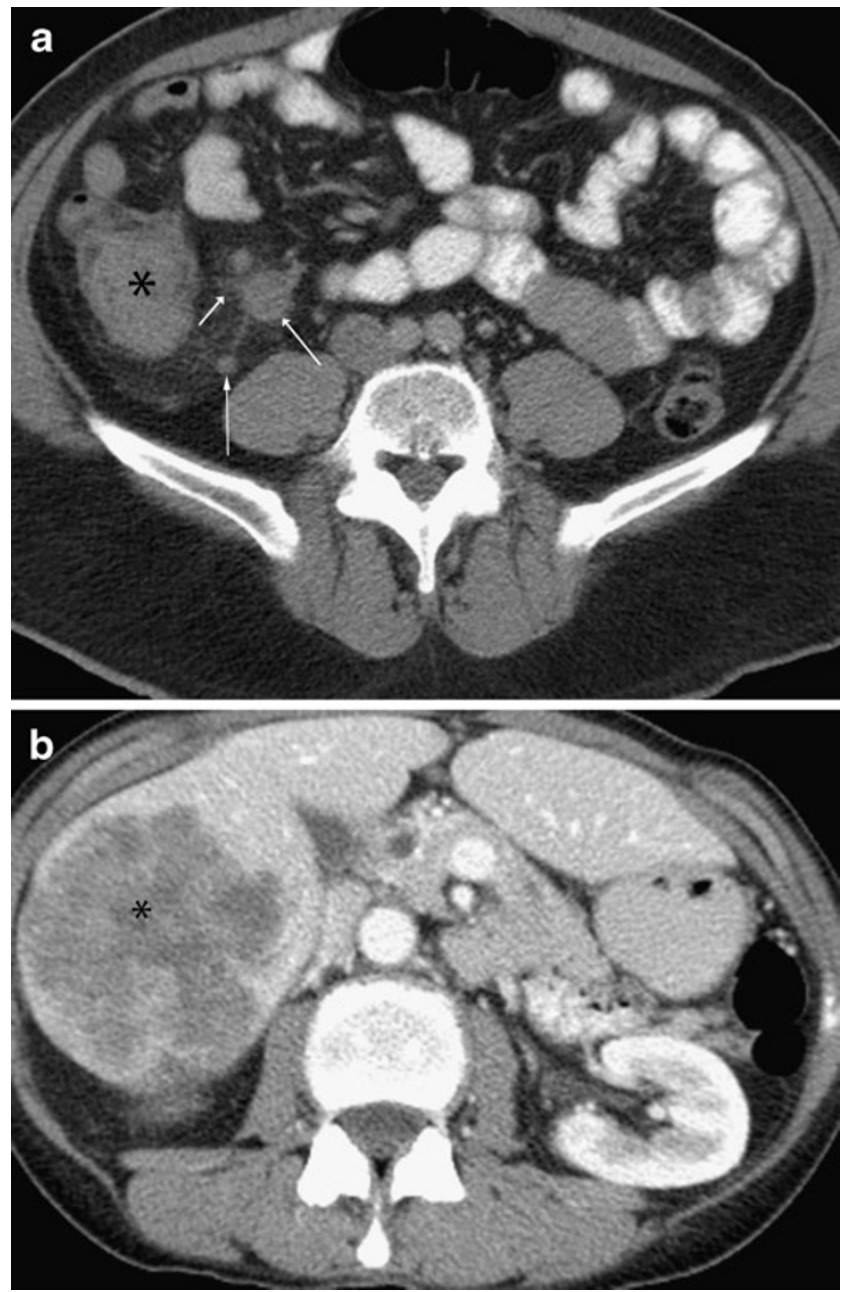

Fig. 4 Colonic adenocarcinoma: a axial non-contrast-enhanced CT showing tumour (asterisk) in the ascending colon with enlarged ileocolic nodes (arrows); b axial contrast-enhanced CT shows a large liver metastasis (asterisk)
Table 3 Summary table of local, nodal and haematogenous spread in colorectal cancer

\begin{tabular}{|c|c|}
\hline Local spread & $\begin{array}{l}\text {-Invasion through the bowel wall into the peri- } \\
\text { colonic fat and adjacent structures }\end{array}$ \\
\hline \multirow[t]{5}{*}{$\begin{array}{l}\text { Lymph node } \\
\text { spread }\end{array}$} & $\begin{array}{l}\text {-Follows the vascular distribution of vessels in } \\
\text { mesocolon; }\end{array}$ \\
\hline & $\begin{array}{l}\text {-Ascending mesocolon: nodes along the ileocolic } \\
\text { vessels and right colic vessel; }\end{array}$ \\
\hline & $\begin{array}{l}\text {-Transverse mesocolon: nodes along the middle } \\
\text { colic vessels; }\end{array}$ \\
\hline & $\begin{array}{l}\text {-Sigmoid and descending mesocolon: nodes along } \\
\text { the inferior mesenteric vein; }\end{array}$ \\
\hline & $\begin{array}{l}\text {-Regional lymph nodes for rectal cancers include: } \\
\text { mesorectal, sigmoid mesenteric, inferior } \\
\text { mesenteric, lateral sacral, presacral, internal } \\
\text { iliac, sacral promontory, superior rectal, middle } \\
\text { rectal and inferior rectal }\end{array}$ \\
\hline $\begin{array}{l}\text { Haematogenous } \\
\text { spread }\end{array}$ & $\begin{array}{l}\text {-Liver (via the portal vein) }>\text { lung, adrenal glands, } \\
\text { bones }\end{array}$ \\
\hline Notes & $\begin{array}{l}\text {-In rectal cancers that have perforated the } \\
\text { peritoneum, transcoelomic spread favours the } \\
\text { lower right small bowel mesentery and the } \\
\text { pouch of Douglas }\end{array}$ \\
\hline
\end{tabular}

and root of the mesentery. Biliary and pancreatic duct dilatation may develop (Fig. 3b). Tumours in the body (Fig. 3c) and tail spread to encase the celiac, hepatic and splenic arteries. Encasement of the proximal superior mesenteric and portal veins can also be seen.

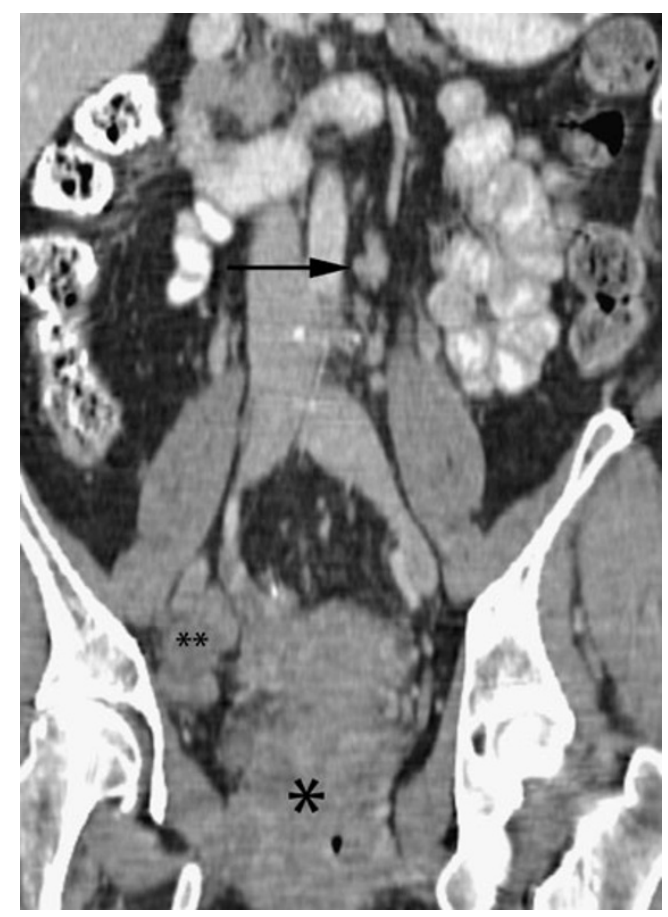

Fig. 5 Rectal adenocarcinoma: coronal reformat CECT showing a bulky rectal tumour (asterisk) with enlarged right internal iliac nodes (double asterisk) and left paraaortic node (arrow) 


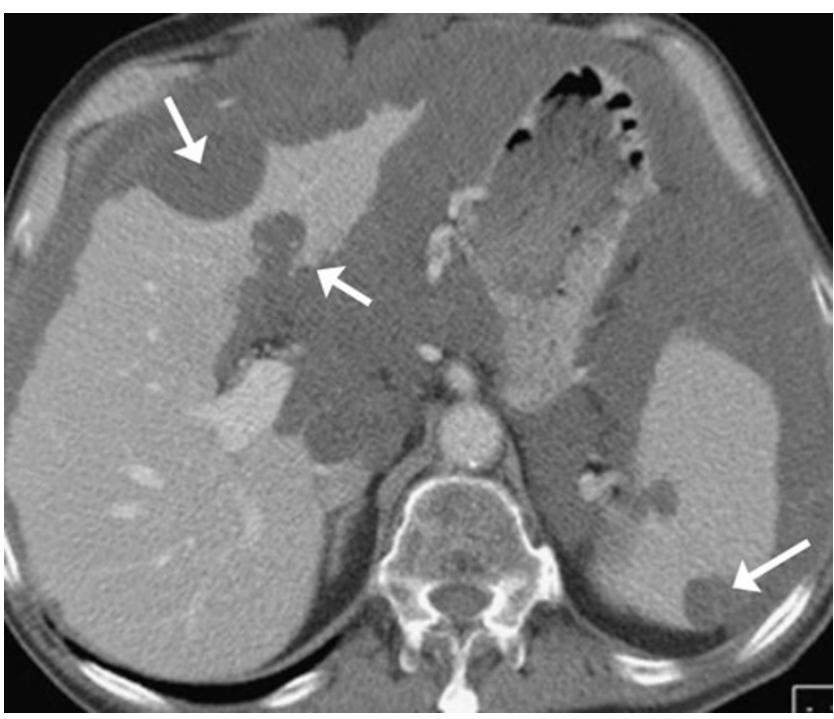

Fig. 6 Pseudomyxoma peritoneii: axial CECT showing mucinous ascites exerting mass effect (scalloping) on the solid organs; primary was of gastrointestinal tract origin

Spread to regional nodes occurs, involving either peripancreatic nodes, nodes at the celiac axis and porta hepatis, or sometimes nodes further afield (Table 2). Haematogeneous spread also arises, with involvement of the liver and peritoneal surfaces. Distant metastases are found in more advanced cases.

\section{Colorectal cancer}

Most colorectal cancers arise from adenomatous polyps, with polyps greater than $2 \mathrm{~cm}$ in size associated with a greater than $40 \%$ risk of malignancy. For local staging of rectal cancer, MRI is now the standard imaging modality because of its increased soft tissue resolution and multiplanar capabilities. Colorectal tumours may appear as polypoid or infiltrative lesions of the bowel wall (Fig. 4a), leading to luminal narrowing and an apple core appearance [9]. Early colonic tumours are better detected when endoluminal distension is given and the colon has been prepared, as in CT colonography. Tumour spread through the serosa appears as pericolonic fat stranding, but may be confused with an associated desmoplastic reaction (possibly mimicking diverticulitis within the sigmoid colon). The presence of fluid in the root of the sigmoid mesentery and

Fig. 7 Metastatic renal cell carcinoma: a coronal reformat CECT showing the inferior vena cava (IVC) greatly expanded by tumour thrombus (arrows) with associated enlarged mediastinal nodes (asterisks). The primary renal tumour has been excised; $\mathbf{b}$ axial CECT showing enhancing lesion in the left gluteus maximus muscle in the same patient, which increased in size on interval CT studies, in keeping with a metastatic deposit; note the numerous venous collaterals in the anterior abdomen (short arrows) due to the IVC thrombus; c axial CECT shows a large lytic deposit in the lumbar spine (asterisk) in the same patient causing compromise of the spinal canal and cord (arrows)
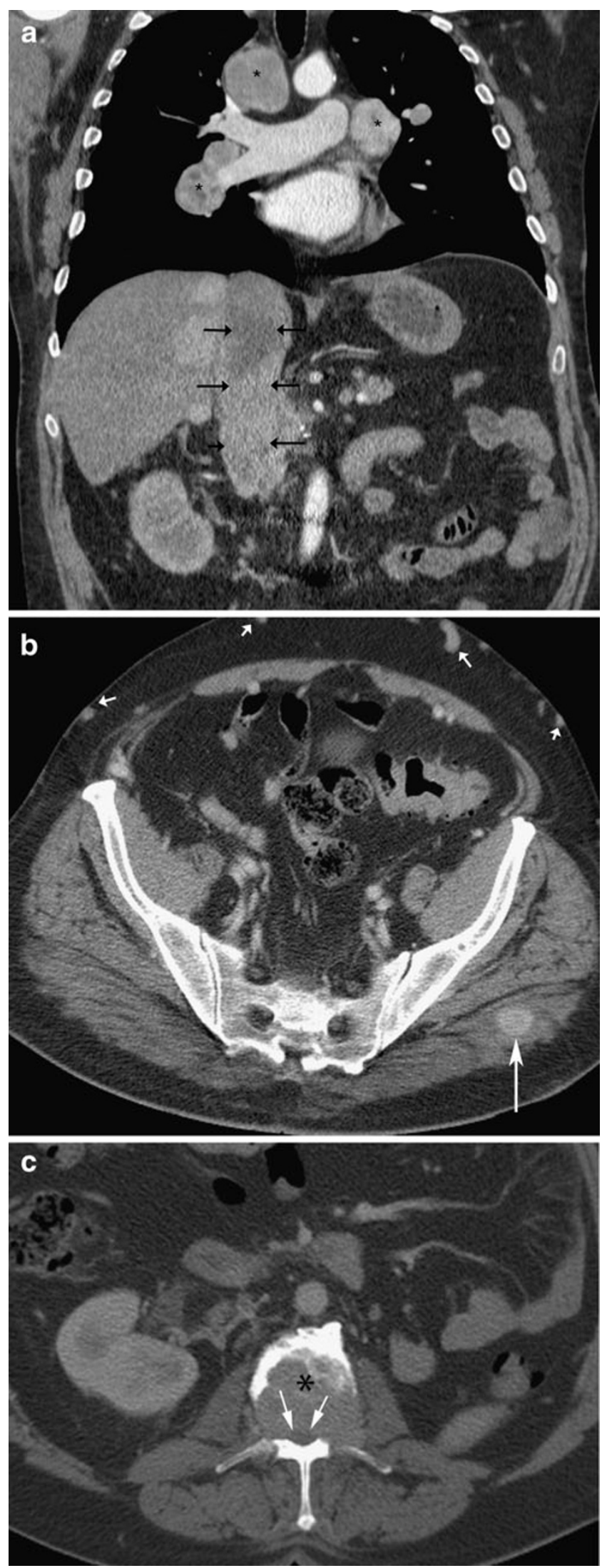
engorgement of the adjacent sigmoid mesenteric vasculature favours diverticulitis, whilst the presence of pericolic lymph nodes is suspicious for colon cancer [10]. Tumour spread to adjacent organs is suggested by loss of the fat planes and direct invasion (see Table 3).

Nodal spread depends on the site of the primary tumour and follows the vascular distribution of the vessels within the mesocolon $[11,12]$. These vessels include the ileocolic vessels and right colic vessel for the ascending mesocolon, the middle colic vessels for the transverse mesocolon, and the inferior mesenteric vein for the sigmoid and descending mesocolon. For rectal tumours, drainage is usually cranial within the mesorectum to involve the regional lymph nodes (Table 3). Involvement of pelvic side wall nodes (Fig. 5) is less common, with involvement of the inguinal nodes only seen with lower rectal tumours when there is proximal lymphatic blockage (e.g. extensive adenopathy).

Metastatic disease at presentation is uncommon $(<10-15 \%$ of cases). Haematogeneous spread is predominantly seen within the liver, with metastases deriving their blood supply from the hepatic artery (compared to normal liver parenchyma, which is primarily supplied by the portal vein). As a result, liver metastases imaged during the portal venous phase are seen as heterogeneous ring-enhancing metastases that are hypodense to the surrounding liver parenchyma (Fig. 4b). If the primary cancer is mucinous, liver metastases may be cystic or calcified. Sites of distant metastases are determined by the venous drainage of the primary site [9]. For example, the venous drainage of the colon and upper rectum is via the portal vein, and thus the liver is a common site of spread. However, the lower rectum has a dual drainage, with the superior haemorrhoidal vein draining into the inferior mesenteric vein and then into the portal vein, and the middle and inferior haemorrhoidal veins draining into the pelvic veins and then directly into the inferior vena cava. This explains why distal rectal cancers can result in isolated pulmonary metastases without hepatic metastases.

Other common sites of metastases include the lungs, adrenal glands and the bones. If rectal tumours perforate the peritoneal membrane, transcoelomic spread may occur, favouring the lower right small bowel mesentery and pouch of Douglas. If colorectal tumours are of mucinous histology, widespread intraperitoneal mucinous metastases may occur with characteristic scalloping of adjacent viscera (pseudomyxoma peritoneii) (Fig. 6).

\section{Renal cell carcinoma (RCC)}

On CT, renal tumours appear as hypervascular masses, with larger lesions of heterogenous attenuation. With increasing size, they spread through the renal capsule into the perinephric fat to involve Gerota's fascia. Early extension through the renal capsule is recognized by an indistinct
Table 4 Summary table of local, nodal and haematogenous spread in renal cell carcinoma

\begin{tabular}{|c|c|}
\hline Local spread & $\begin{array}{l}\text {-Perinephric fat; ipsilateral adrenal; adjacent } \\
\text { viscera (including muscles); } \\
\text { •Renal vein invasion ( } \pm \text { IVC) }\end{array}$ \\
\hline $\begin{array}{l}\text { Lymph node } \\
\text { spread }\end{array}$ & $\begin{array}{l}\text {-Via lymphatics following the renal vessels to the } \\
\text { ipsilateral para-aortic nodes; direct connections } \\
\text { with the thoracic duct and mediastinum also } \\
\text { exist }\end{array}$ \\
\hline $\begin{array}{l}\text { Haematogenous } \\
\text { spread }\end{array}$ & -Common sites: lungs $>$ bones, CNS, adrenals \\
\hline Note & $\begin{array}{l}\text {-Extension into renal vein occurs in } 20 \% \text { of } \\
\text { patients at presentation; IVC involvement in } \\
5-10 \%\end{array}$ \\
\hline
\end{tabular}

tumour margin, thickened perirenal fascia and perinephric fat stranding [13]. Extension into the renal vein occurs in $20 \%$ of patients at presentation with involvement of the inferior vena cava (IVC) in $5-10 \%$ of patients (Fig. 7a) [13]. This is more likely with larger tumours. Tumour thrombus is seen as a filling defect within the vein, which should not be confused with streaming artefact from unopacified blood. The presence of venous distension on its own may be misleading as RCCs are hypervascular and hence tend to have increased blood flow and venous drainage. Distinction between tumour thrombus and bland thrombus within the IVC may be difficult, although the former may have enhancing tumour vessels within it. In advanced tumours, direct invasion into adjacent structures such as the diaphragm, and posterior abdominal wall muscles may be seen (see Table 4).

Lymphatic spread from RCC tends to follow the renal veins to involve the ipsilateral para-aortic nodes. There are

Table 5 Summary table of local, nodal and haematogenous spread in ovarian carcinoma

\begin{tabular}{|c|c|}
\hline Local spread & $\begin{array}{l}\text {-Uterus and broad ligament (via the fallopian } \\
\text { tube); } \\
\text {-Direct invasion of the rectum, colon, bladder and } \\
\text { pelvic side wall }\end{array}$ \\
\hline $\begin{array}{l}\text { Lymph node } \\
\text { spread }\end{array}$ & $\begin{array}{l}\text {-Via lymphatics travelling along with the ovarian } \\
\text { vessels to terminate in common iliac and para- } \\
\text { aortic nodes; } \\
\text {-Via the broad ligament to terminate in the } \\
\text { internal iliac and obturator nodes; } \\
\text {-Via the round ligament to terminate in the } \\
\text { external iliac and inguinal nodes }\end{array}$ \\
\hline $\begin{array}{l}\text { Trans-coelomic } \\
\text { spread }\end{array}$ & $\begin{array}{l}\text { - Common sites: undersurface of the diaphragm, } \\
\text { liver surface, pouch of Douglas, omentum, } \\
\text { serosal bowel surfaces }\end{array}$ \\
\hline $\begin{array}{l}\text { Haematogenous } \\
\text { spread }\end{array}$ & $\begin{array}{l}\text {-This occurs late during the disease; } \\
\text {-Liver>lungs, kidney, bone }\end{array}$ \\
\hline Note & $\begin{array}{l}\text {-Ascites arise from increased production from } \\
\text { tumour surfaces and/or occlusion of } \\
\text { retroperitoneal lymph nodes }\end{array}$ \\
\hline
\end{tabular}



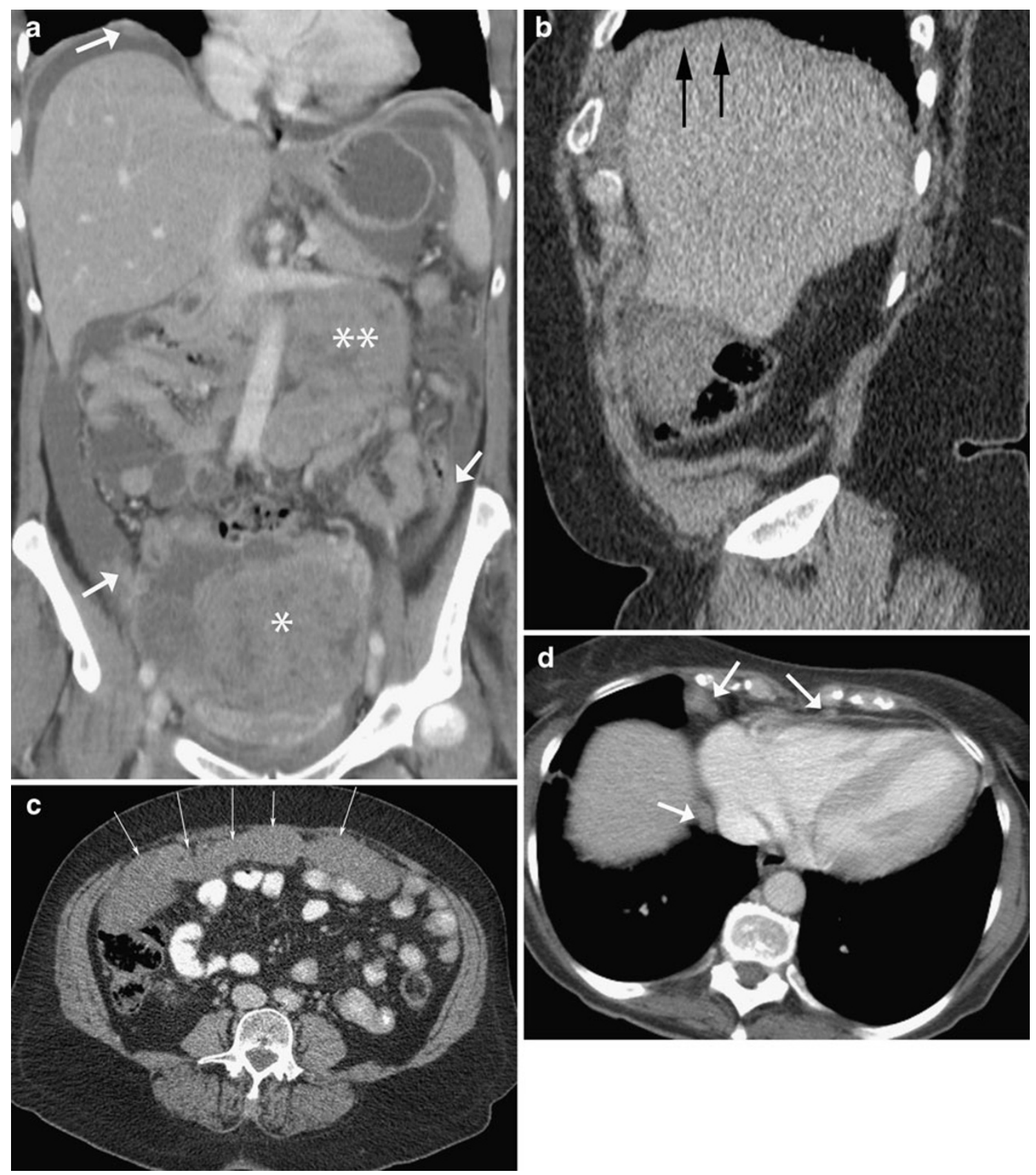

Fig. 8 Ovarian carcinoma: coronal reformat CECT showing a large solid/cystic adnexal mass arising from the pelvis (asterisk), with largevolume para-aortic adenopathy (double asterisk), ascites and small peritoneal deposits (arrows). Although para-aortic nodal involvement is seen in metastatic ovarian carcinoma, the large volume is rather

unusual; b sagittal reformat CECT showing subcapsular deposits over the liver in a different patient (arrows); $\mathbf{c}$ axial CECT in the same patient as (b) showing a thick omental cake (arrows); d axial CECT in the same patient as (b) showing multiple enlarged (short axis diameter $>5 \mathrm{~mm}$ ) cardiophrenic nodes (arrows)

Table 6 Summary table of local, nodal and haematogenous spread in prostate cancer

$\begin{array}{ll}\text { Local spread } & \cdot \text { Direct extension through the prostate capsule into the seminal vesicles and bladder base } \\ \text { Lymph node spread } & \cdot \text { Order of nodal involvement: obturator, presacral, internal iliac, common iliac } \\ \text { Haematogenous } & \cdot \text { Bone }>>\text { lung and liver; } \\ \text { spread } & \cdot \text { Spinal bone metastases are the commonest site (due to the direct communication between the presacral and periprostatic veins) } \\ \text { Notes } & \cdot \text { Denonvillier's fascia forms a relative natural barrier to rectal spread; } \\ & \cdot \text { Tumours at the apex of the prostate are more likely to demonstrate extracapsular extension because of relatively little capsule } \\ & \text { at this level }\end{array}$



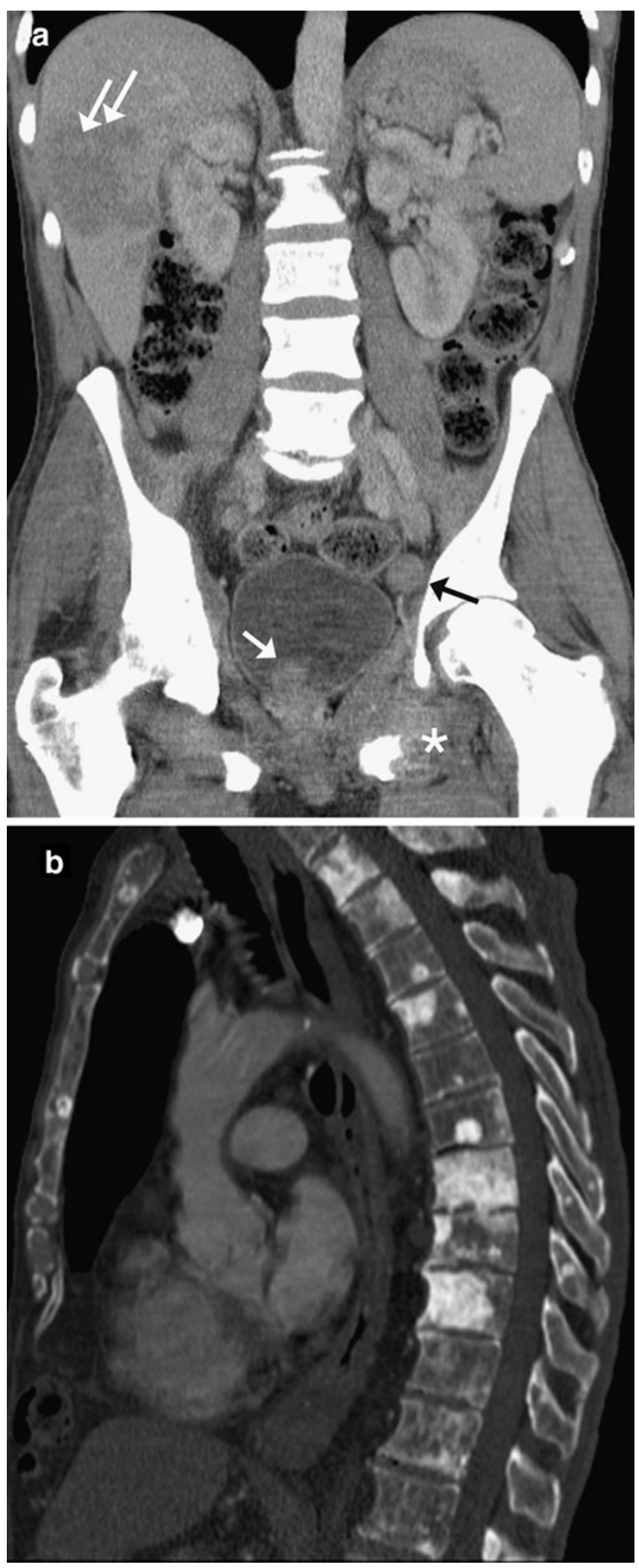

also direct connections with the thoracic duct and mediastinum, which can account for the rare presence of mediastinal and hilar node involvement (Fig. 7a) at presentation (especially on the right side).
Fig. 9 Prostate carcinoma: a coronal reformat CECT showing irregular enlarged prostate tumour (white arrow) extending into the bladder with enlarged left pelvic side wall node (black arrow), liver metastases (double arrows) and lesion in the left pubic ramus with large soft tissue component $(*)$. Note the bones are sclerotic in keeping with diffuse bony metastases; $\mathbf{b}$ sagittal reformat CECT in a different patient on bone windows, showing multiple sclerotic metastases in the thoracolumbar spine

Between $25-35 \%$ of patients with RCC have metastases at diagnosis [14]. Metastatic involvement in renal cell carcinoma is seen (in order of decreasing frequency) in: the lung $(50-60 \%)$, bone $(30-40 \%)$, liver (30-40\%), adrenal gland (5\%), contralateral kidney (5\%), retoperitoneum (5\%) and brain (5\%) [14]. More unusual sites that have been reported include the pancreas, peritoneum, bowel, thyroid and muscle (Fig. 7b) and subcutaneous tissues. Visceral metastases tend to be hypervascular, similar to the primary tumour. Bony involvement appears as expansile lytic lesions within the axial skeleton (Fig. 7c).

\section{Ovarian carcinoma}

Cross-sectional imaging (CT or MRI) is used for preoperative tumour assessment, with $\mathrm{CT}$ being the imaging modality of choice for assessing metastatic disease. Bowel opacification is usually achieved with a positive oral contrast medium (e.g. dilute gastrograffin), which is essential for the detection of peritoneal deposits. Intravenous contrast is also routinely administered unless contraindicated, with imaging performed in the portal venous phase. Conversely, local pelvic spread is better assessed with pelvic MRI than CT (see Table 5).

Primary ovarian cancer has variable appearances, presenting either as a solid mass or mixed solid/cystic lesion (Fig. 8a). If predominantly cystic, internal septations and/or mural nodules are seen. Ovarian cancer spreads locally [15] to adnexal structures, the uterus and the contralateral ovary, although bilateral ovarian cancer may occur in $11-50 \%$ of cases. Invasion of the pelvic side wall is indicated when the tumour is seen encasing the iliac vessels or is within $3 \mathrm{~mm}$ of the pelvic sidewall [16]. Direct infiltration of the rectosigmoid colon or bladder wall may be seen.

Peritoneal dissemination is the commonest mode of spread, being found in approximately $70 \%$ of patients at presentation. Common sites include the greater omentum, paracolic gutters, the pouch of Douglas, the liver capsule (Fig. 8b), the diaphragm and bowel serosa. Less commonly, implants may be seen within the mesentery, along the porta hepatis, lesser sac, splenic surface and gastrosplenic ligament [16-18]. Ascites are often present, due to either increased production from tumour surfaces or reduced peritoneal resorption from invasion of lymphatics by tumour cells. Peritoneal deposits on CT have a variety of 
appearances including enhancing nodular soft tissue lesions, linear/plaque-like thickening of peritoneal reflections (Fig. 8a), thick omental cakes (Fig. 8c), tiny calcifications or mixed solid and cystic or purely cystic lesions $[19,20]$. The involvement of bowel serosa can lead to (asymmetric) bowel wall thickening, tethering of bowel loops and bowel obstruction. Liver surface implants (subcapsular deposits) may be seen, with deposits causing characteristic scalloping of the liver capsule. Metastatic mucinous tumours of the ovary can lead to pseudomyxoma peritoneii, although in many of these cases, the primary mucinous tumour actually arises from the gastrointestinal tract with secondary involvement of the ovary.

Lymphatic spread occurs via three routes and is usually seen in conjunction with peritoneal spread. Routes include: (1) along the ovarian vessels to the common iliac and paraaortic nodes (Fig. 8a); (2) via the broad ligament and parametria to the internal iliac and obturator nodes; and (3) rarely via the round ligaments to the external iliac and inguinal nodes. Cardiophrenic nodes (defined as $>5 \mathrm{~mm}$ short axis diameter on CT) [21] are characteristically involved as they are the main lymphatic drainage route from the peritoneal cavity (Fig. 8d).

Haematogenous dissemination is rare in ovarian carcinoma with a malignant pleural effusion being the most common manifestation. At presentation, liver metastases are seen in less than $1 \%$ of cases. Other sides of spread include the spleen, kidneys, adrenals, lungs, brain and bone.

\section{Prostate carcinoma}

MRI is traditionally used for local staging of prostate cancer because of its high contrast resolution (as in rectal cancer). It is able to differentiate organ-confined disease from tumour that demonstrates extracapsular spread. Direct involvement of the seminal vesicles or adjacent organs is also well seen on MRI. CT is used for nodal and distant staging of prostate cancer (see Table 6).

Classically, two lymph node metastatic patterns have been reported [22]. Pattern 1 includes metastatic spread to pelvic (Fig. 9a) and para-aortic lymph nodes, and this pelvic nodal involvement is sequential with spread to the obturator nodes, presacral nodes, internal iliac and common iliac nodes. Pattern 2 involves para-aortic nodes only, with this second pattern of nodal spread felt to be due to haematogenous dissemination.

Distant spread occurs in one third of patients with involved sites including bone $(90 \%)$, lung $(50 \%)$, liver (25\%) (Fig. 9a), pleura (21\%) and adrenals (13\%) [23]. Osteoblastic sclerotic metastases rather than lytic bone lesions are usually seen on CT. In order of decreasing frequency, skeletal metastases are seen within the vertebrae (Fig. 9b), sternum, pelvic bones, ribs and femora. Spinal involvement is initially more common within the lumbar region and less likely within the cervical spine $(97 \%$ cf. $38 \%$ ), suggestive of upward metastatic spread along the spinal veins [23]. Pulmonary metastases occur in between $5-27 \%$ of patients at presentation [24] and are more often seen in association with the second pattern of nodal involvement [22]. Pulmonary involvement can either appear as lymphangitis carcinomatosa (due to direct invasion of the pulmonary lymphatics) or as pulmonary nodules (due to haematogenous spread) [22, 24].

\section{Conclusion}

This review has described the typical patterns of tumour spread for the more common cancers occurring within the abdomen and pelvis. Sites of nodal and haematogenous dissemination clearly vary depending on tumour type. Knowledge of the characteristic sites of spread in such cancers is felt to be essential for the radiologist when reporting staging and restaging $\mathrm{CT}$ scans. It is hoped that the summary tables in particular will act as a useful aidemémoir to the reporting radiologist. Correct identification of metastatic sites of disease is important, as this will not only have an impact on patient management, but also a bearing on patient prognosis.

\section{References}

1. Crew KD, Neugut AI (2006) Epidemiology of Gastric cancer. World J Gastroenterol 12(3):354-362

2. Shimizu K, Ito K, Matsunaga N, Shimizu A, Kawakami Y (2005) Diagnosis of gastric cancer with MDCT using the water-filling method and multiplanar reconstruction: CT-histologic correlation. AJR Am J Roentgenol 185(5):1152-1158

3. Rossi M, Broglia L, Graziano P, Maccioni F, Bezzi M, Masciangelo R, Rossi P (1999) Local invasion of gastric cancer: $\mathrm{CT}$ findings and pathologic correlation using 5-mm incremental scanning, hypotonia, and water filling. AJR Am J Roentgenol 172 (2):383-388

4. McLoughlin JM (2004) Adenocarcinoma of the stomach: a review. Proc (Bayl Univ Med Cent) 17(4):391-399

5. Chourmouzi D, Sinakos E, Papalavrentios L, Akriviadis E, Drevelegas A (2009) Gastrointestinal stromal tumors: a pictorial review. J Gastrointestin Liver Dis 18(3):379-383

6. Hong X, Choi H, Loyer EM, Benjamin RS, Trent JC, Charnsangavej C (2006) Gastrointestinal stromal tumor: role of CT in diagnosis and in response evaluation and surveillance after treatment with imatinib. Radiographics 26:481-495

7. Lall CG, Howard TJ, Skandarajah A, DeWitt JM, Aisen AM, Sandrasegaran K (2007) New concepts in staging and treatment of locally advanced pancreatic head cancer. AJR Am J Roentgenol 189(5):1044-1050

8. Vikram R, Balachandran A, Bhosale PR, Tamm EP, Marcal LP, Charnsangavej C (2009) Pancreas: peritoneal reflections, ligamentous connections, and pathways of disease spread. Radiographics 29(2):e34 
9. Horton KM, Abrams RA, Fishman EK (2000) Spiral CT of colon cancer: imaging features and role in management. Radiographics 20(2):419-430

10. Chintapalli KN, Esola CC, Chopra S, Ghiatas AA, Dodd GD III (1997) Pericolic mesenteric lymph nodes: an aid in distinguishing diverticulitis from cancer of the colon. AJR Am J Roentgenol 169:1253-1255

11. McDaniel KP, Charnsangavej C, DuBrow RA, Varma DG, Granfield CA, Curley SA (1993) Pathways of nodal metastasis in carcinomas of the cecum, ascending colon, and transverse colon: CT demonstration. AJR Am J Roentgenol 161:61-64

12. Granfield CA, Charnsangavej C, DuBrow RA et al (1993) Regional lymph node metastases in carcinoma of the left side of the colon and rectum: CT demonstration. AJR Am J Roentgenol 159:757-761

13. Reznek RH (2004) CT/MRI in staging renal cell carcinoma. Cancer Imaging. 4 Spec No A:S25-32

14. Griffin N, Gore ME, Sohaib SA (2007) Imaging in metastatic renal cell carcinoma. AJR Am J Roentgenol 189(2):360-370

15. Forstner $R$ (2007) Radiological staging of ovarian cancer: imaging findings and contribution of CT and MRI. Eur Radiol 17(12):32233235

16. Forstner R, Hricak H, Occhipinti KA, Powell CB, Frankel SD, Stern JL (1995) Ovarian cancer: staging with CT and MRI. Radiology 197:619-626
17. Tempany CM, Zou KH, Silverman SG, Brown DL, Kurtz AB, McNeil BJ (2000) Staging of advanced ovarian cancer: comparison of imaging modalities - report from the Radiology Oncology Group. Radiology 215:761-767

18. Coakley FV (2002) Staging ovarian cancer: role of imaging. Radiol Clin N Am 40:609-636

19. Forstner R (2006) CT and MRI in ovarian carcinoma. In: Hamm B, Forstner R (eds) CT and MRI of the female pelvis. Springer, Berlin Heidelberg New York, pp 231-261

20. Mitchell DG, Hill MC, Hill S, Zaloudek C (1986) Serous carcinoma of the ovary: CT identification of metastatic calcified implants. Radiology 158:649-652

21. Holloway BJ, Gore ME, A'Hern RP, Parsons C (1997) The significance of paracardiac lymph node enlargement in ovarian cancer. Clin Radiol 52:692-69

22. Saitoh H, Yoshida K, Uchijima Y, Kobayashi N, Suwata J, Kamata S (1990) Two different lymph node metastatic patterns of a prostate cancer. Cancer 65:1843-1846

23. Bubendorf L, Schöpfer A, Wagner U, Sauter G, Moch H, Willi N, Gasser TC, Mihatsch MJ (2000) Metastatic patterns of prostate cancer: an autopsy study of 1,589 patients. Hum Pathol 31 (5):578-583

24. Fabozzi SJ, Schellhammer PF, el-Mahdi AM (1995) Pulmonary metastases from prostate cancer. Cancer 75:2706-2709 